\title{
USING TRANSFER LEARNING FOR VIDEO POPULARITY PREDICTION
}

\author{
Akshatha M R $\mathbf{R}^{1}$, Prathima V $\mathbf{R}^{\mathbf{2}}$ \\ ${ }^{I} P G$ Student, Dept. of Computer Science and Engineering, RGIT, Karnataka, India \\ ${ }^{2}$ Assistant Professor, Dept. of Computer Science and Engineering, RGIT, Karnataka, India
}

\begin{abstract}
Knowledge gained from social streams can be used to address many multimedia problems which cannot be solved by using traditional multimedia techniques alone. Some portion of videos in video portals exhibit sudden (bursty) rise in popularity, an effect which is not captured by video domain features alone Cross domain real-time transfer learning framework is used which utilizes knowledge from social streams (e.g., Twitter) and improve popularity prediction in the video domain. OSLDA model is used to detect topics from social streams [3]. Social Transfer algorithm is used for classifying videos with topics which is then used to calculate the social prominence and finally leading to the improved popularity prediction in the video domain. The framework has the ability to scale with incoming tweets in real time.
\end{abstract}

Keywords - Cross-domain media retrieval, Topic model, Twitter, Video popularity, YouTube

\section{INTRODUCTION}

Knowledge from social streams is used to solve many multimedia problems that could not be solved only by using traditional techniques. Social microblogs like Twitter is used to capture real time events faster because of its short text format [3]. Thus any real world event first originates in twitter and then it is searched online, which finally leads to increased views for videos related to that event.

Social streams and traditional media are unevenly distributed on the internet depending on platform, popularity and bias [5]. Potential is limited within the domain where it resides. To predict sudden rise in popularity of videos we need to incorporate cross-domain information transfer mechanism which transfers information gained from social streams (Twitter) to video streams (YouTube).

There exist some challenges in performing cross-domain popularity predictions using social streams.

- Knowledge cannot be directly transferred from one domain to another because classification patterns and data distributions are difficult among domains.

- It is challenging to extract relevant information from social streams like Twitter because of its noisy and short form nature.

- An algorithm which can scale to the real-time nature of the social streams.

Social media from disparate sources on the internet can be connected by building a common topic space. In particular Twitter is considered as the social stream and videos collected from a video search engine is considered to be social media in this work. OSLDA model is used to extract and update topic space in real time. Topic space acts like a bidirectional bridge between the video domain and twitter domain. Social Transfer algorithm allows for classifying videos with topics by using topic space [7]. The algorithm also allows for continuos updating the topic space and integration of fresh topics as newer tweet is encountered. Social prominence of each topic is calculated. Videos with social prominence will demonstrate sudden/bursty rise in popularity.

\section{SOCIAL TRANSFER}

In Social Transfer, we have considered two datasets in the target domain: the training data set with labels and the test data set without labels which contains $\mathrm{M}$ and $\mathrm{N}$ instances respectively. Source domain consist of auxiliary data set which contains K tweets instances. The Single Transfer framework used for this purpose is represented as a transfer graph which is shown in fig 1 . In cross-domain learning the auxiliary data and the target data share the same categories but are in different domains [4]. Cross domain learning to predict video popularity includes following steps: ( A ) learning topics from social streams ( B ) mapping the relationships among the auxiliary and target data by constructing the transfer graph ( C ) online spectral learning of the transfer graph.

\subsection{Learning Topics From Social Streams}

OSLDA model is used to extract and learn topics from social streams populate and update topics in the topic space scaling with streaming tweets in real time. The reason behind building a topic space is to construct a platform upon which multiple 
media applications can be forged. Topic space acts like a bidirectional bridge between tweets and videos. OSLDA model is used to enrich tweets with relevant video recommendations and empowering videos with social popularity estimated from social stream.

\subsection{Transfer Graph}

Transfer learning makes use of the available auxiliary data to help the learning on the target data. Auxiliary data and the target data share some common relations between them. We can model the relations between auxiliary data and target data by constructing a Transfer graph. The main purpose of transfer graph is to capture the cross domain attributes of social streams and videos in the transfer learning task. The transfer graph G ( V , E ) consists of vertices representing instances, features and labels while edges denotes the relations between the end nodes, connecting the auxiliary and target data.

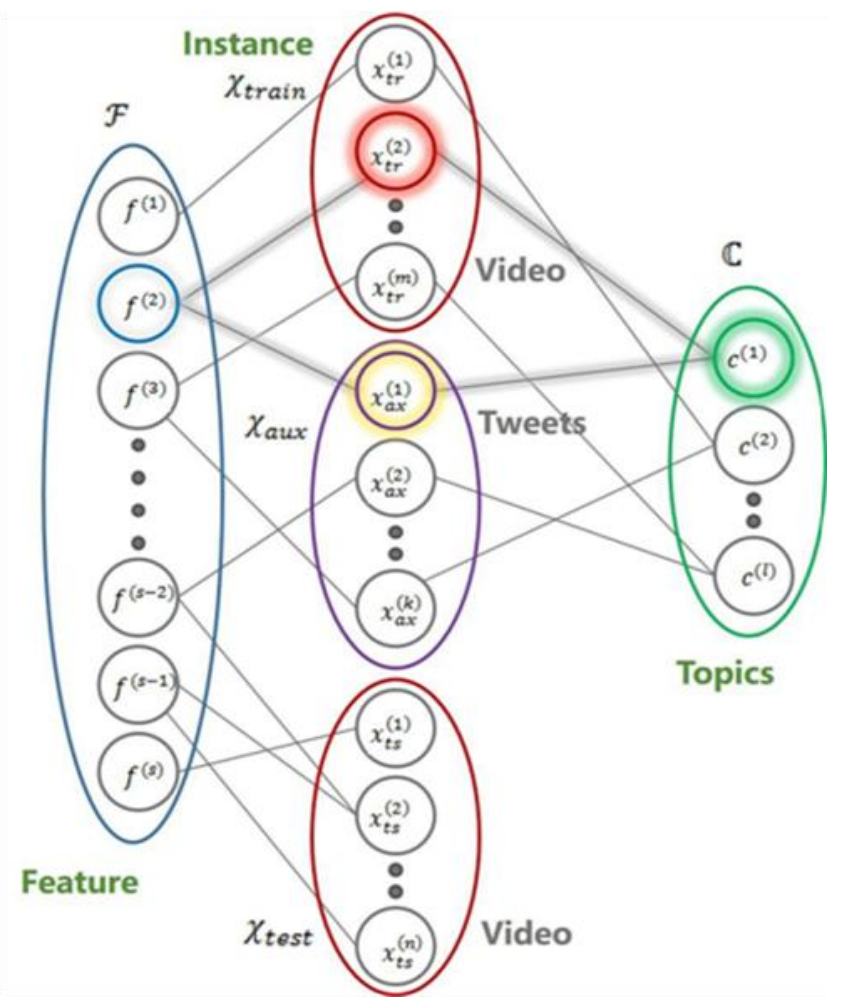

Fig-1: Transfer graph for social transfer

\subsection{Spectral Learning of the Transfer Graph}

Spectral analysis of the transfer graph G ( V, E ) is used to extract eigen vectors, which combines the principal component features from the training and auxiliary data.

Traditional spectral classification methods focused only on the scenario that, the labeled data and the unlabeled data are drawn from the same domain. But, unfortunately many scenarios in the real world do not follow this requirement. In many real world applications, we make use of the labeled data from one domain to classify the unlabeled data in a different domain. Obtaining labeled data in one domain is difficult while there are plenty of labeled data from a related but different domain [6]. Previous approaches in transfer learning have suggested the use of normalized cut technique for eigen vector extraction [4]. But, since the normalized cut technique is incapable of scaling with the twitter stream, Power Iteration technique can be used for eigen vector extraction [5]. Power Iteration technique is very fast on large datasets, running over 1,000 times faster than an NCut technique [8].

The process of extracting eigen vectors from the Transfer graph using Power Iteration technique is as shown in the fig 2. To extract top q eigen vectors of the transfer graph $\mathrm{G}=(\mathrm{V}, \mathrm{E}$ ), the graph must be converted into a Laplacian matrix. Since the topics are updated with time, we need to progressively incorporate these new topics into the transfer graph, which in effect recalculates the weights of edge/path between the features and the corresponding labels within the transfer graph. Rank update on the Laplacian Matrix is performed which adds negative weights between feature words that belong to different topics and adds positive weights between feature words that share the same topic. Rank update on the Laplacian Matrix can improve eigenvector extraction speed by spreading the eigen gap. Once the first q eigenvectors have been found by iteratively using the Power Iteration technique, we can form a combined feature representation that depends on both the auxiliary data and the training data. Traditional classification algorithm like SVM can then be used to train the classifier and then classify the test data in the eigen feature space.

\section{SOCIAL POPULARITY}

Social Transfer is used in Socialized Query Suggestion for Video Searching and Video Recommendation [7]. Social Transfer can also be used for predicting social popularity of videos. The steps involved are: ( A ) finding social prominence of a video and estimating trend aware popularity ( B ) predicting which videos will demonstrate bursty nature.

\subsection{Finding Social Prominence and Estimating TAP}

Trends remain socially prominent for a certain period of time, after which they suffer inevitable decay. Finding social prominence of a video requires calculating the trending score ( Tscore ) [5]. For some video v1, let a1 be the topic to which the video has maximum membership. Using Social Transfer Classification the membership measure can be easily retrieved. Then the social prominence of video v1 is Tscore a1.

Trend Aware Popularity ( TAP ) can be obtained by fusing traditional popularity of a video ( based on its view counts ) and its social prominence. In a traditional video ranking system like in You Tube, the videos in the recommended list 
get clicked more often resulting in subsequent higher view counts for them [1]. Therefore it is reasonable to fuse social prominence of a video with its traditional popularity. This fusion of social prominence of the video and traditional popularity factors like view counts is called TAP [2].

\subsection{Predicting Bursty Videos}

If a topic is trending in the social domain, then the video belonging to the same topic will gain popularity in other domains. TAP score reflects the social popularity as well as the traditional popularity of a video. The ratio of TAP to the Tscore value will give us the impact of the social signal in boosting the video popularity. If the value obtained is low, then the video has high impact of social prominence in comparison to the adjusted view count score. Thus for a certain video, if this ratio is significantly lower than for others, we predict the video will gain bursty popularity.

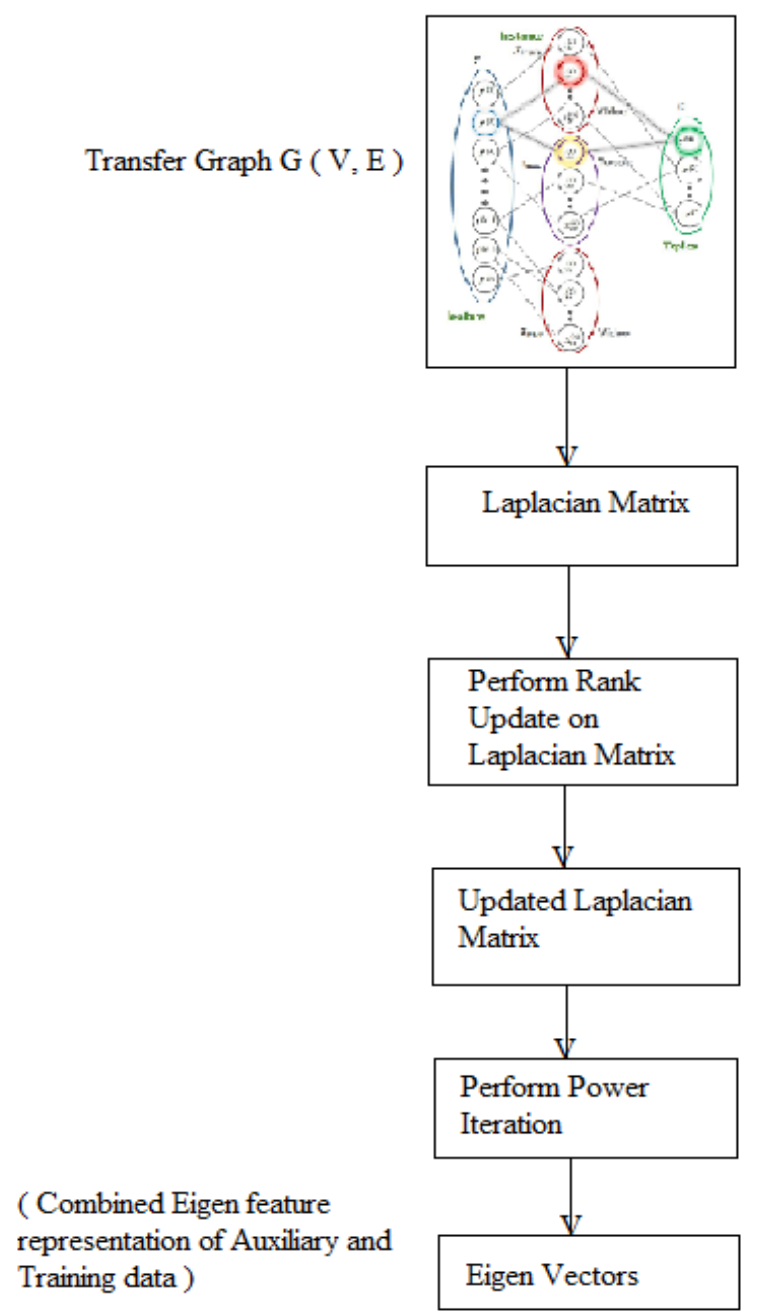

Fig-2: The process of extracting eigen vectors from the Transfer graph using Power Iteration technique.

\section{CONCLUSIONS}

This paper presents a novel cross-domain real time transfer learning approach based on social streams called Social Transfer. We demonstrate the use of Social learning in predicting the video popularity. Social Transfer can be applied to various multimedia applications which can be boosted by the knowledge acquired from cross-domain social data. Video only possesses tags entered by the user or extracted from the title. Tag enrichment by visual object understanding or by comment extraction will improve the prediction power of the model.

\section{REFERENCES}

[1]. R. Zhou, S. Khemmarat, and L. Gao, "The impact of youtube recommendation system on video views," in Proc. Int. Conf. Internet Measurement, 2010.

[2]. K. Filippova and K. B. Hall, "Improved video categorization from text metadata and user comments," in Proc. ACM SIGIR Conf. Res. and Develop. in Inf. Retrieval, 2011.

[3]. S. D. Roy, T. Mei, W. Zeng, and S. Li, "Empowering cross-domain Internet media with real-time topic learning from social streams," in Proc. IEEE Int. Conf. Multimedia and Expo, 2012.

[4]. W. Dai, O. Jin, G. Xue, Q. Yang, and Y. Yu, "Eigentransfer: a unified framework for transfer learning," in Proc. Int. Conf. Mach. Learning, 2009.

[5]. S. D. Roy, T. Mei, W. Zeng, and S. Li, "Towards crossdomain learning for social video popularity prediction," in IEEE Transactions on multimedia, 2013.

[6]. X. Ling, W. Dai, G.-R. Xue, Q. Yang, and Y. Yu, "Spectral domain transfer learning," in Proc. ACM Conf. Knowledge Discovery and Data Mining, 2008

[7]. S. R. Deb, T. Mei, W. Zeng, and S. Li, "Socialtransfer: Cross-domain transfer learning from social streams for media applications," in Proc. ACM Multimedia, 2012.

[8]. F. Lin and W. W. Cohen, "Power iteration clustering," in Proc. Int. Conf. Mach. Learning, 2010.

[9]. [Online]. Available: http://trec.nist.gov/data/tweets/. 